\title{
Treatment of Traumatic Esophagopleural Fistula Using the Over-the-Scope-Clip System
}

\author{
Ji Hyoung Kim¹, Jong-Jae Park', II Woo Jung ${ }^{1}$, Sang Hoon Kim¹, Hee Dong Kim¹, Jung Wan Choe', Moon Kyung Joo' and Hyun Gu Kim² \\ ${ }^{1}$ Division of Gastroenterology, Department of Internal Medicine, ${ }^{2}$ Department of Cardiothoracic Surgery, Korea University Guro Hospital, \\ Korea University College of Medicine, Seoul, Korea
}

Esophagopleural fistula (EPF) is a rare condition that is usually accompanied by severe infection and life-threatening morbidity. Here, we report the successful treatment of an EPF by closing an esophageal orifice using the over-the-scope-clip (OTSC) system without postprocedural complications. A 41-year-old man had serious thoracic and abdominal trauma due to a traffic accident. Computed tomography revealed findings suggestive of esophageal rupture due to Boerhaave syndrome. An emergent explorative operation was performed for primary repair with the insertion of a vacuum-assisted closure device. A postoperative upper gastrointestinal series revealed an EPF tract connecting the left pleural space and distal esophagus. We performed an endoscopic procedure using the "traumatic-type" OTSC to seal the EPF, and the esophageal orifice was completely healed 2 weeks postoperatively. The OTSC system might represent a safe and feasible modality for the treatment of EPF. Clin Endosc 2015;48:440-443

Key Words: Esophagopleural fistula; Over-the-scope-clip; Negative-pressure wound therapy

\section{INTRODUCTION}

Esophagopleural fistula (EPF) is a rare postoperative complication that commonly occurs after pneumonectomy. However, it sometimes develops after other traumatic events, such as traffic accidents. EPF is usually accompanied by serious infection and life-threatening morbidity. Although various endoscopic or surgical techniques have been developed for the treatment of EPF, EPF-related mortality remains high. ${ }^{1}$

The vacuum-assisted closure (VAC) device (KCI Medical, San Antonio, TX, USA) was first described in 1997 for wound healing. ${ }^{2}$ The device has four mechanisms of action: (1) contraction of the wound, (2) stabilization of the wound, (3) removal of extracellular fluid, and (4) microdeformation

Received: October 24, 2014 Revised: October 29, 2014

Accepted: October 29, 2014

Correspondence: Jong-Jae Park

Division of Gastroenterology, Department of Internal Medicine, Korea University Guro Hospital, Korea University College of Medicine, 148 Gurodong-ro, Gurogu, Seoul 08308, Korea

Tel: +82-2-2626-1771, Fax: +82-2-853-1943, E-mail: gi7pjj@korea.ac.kr

cc This is an Open Access article distributed under the terms of the Creative Commons Attribution Non-Commercial License (http://creativecommons.org/ licenses/by-nc/3.0) which permits unrestricted non-commercial use, distribution, and reproduction in any medium, provided the original work is properly cited. of the wound surface. ${ }^{2}$ The VAC device reduces bacterial levels and improves wound healing and surgical results. ${ }^{3}$ Thus, this procedure has been applied to various types of chronic non-healing wounds, including esophageal and intra-abdominal injuries. However, VAC application is not always effective for complete healing of more complicated conditions such as enterocutaneous fistula. ${ }^{4}$

The over-the-scope-clip (OTSC) system (Ovesco Endoscopy AG, Tübingen, Germany) is a newly designed endoscopic method for the mechanical compression of large areas in the gastrointestinal (GI) tract. Currently, the OTSC is used for hemostasis, ${ }^{5}$ for closure of iatrogenic full-thickness perforations during endoscopic resection, ${ }^{6}$ or after natural orifice transluminal endoscopic surgery (NOTES), ${ }^{7}$ and the OTSC system has been reported to be effective, safe, and easy to use for the management of bleeding and leakages from perforations of the GI tract without major complications. ${ }^{8,9}$ Furthermore, a recent study showed that a gastric fistula could be successfully closed using the OTSC system. ${ }^{10}$

Here, we report the successful treatment of an EPF refractory to VAC application by closure of an esophageal orifice using the OTSC system without postprocedural complications. 


\section{CASE REPORT}

A 41-year-old man had major thoracic and abdominal trauma due to a serious traffic accident in which he was buried under a heavy crane. Computed tomography revealed pleuroparenchymal injury (pneumothorax, pleural effusion, and subcutaneous emphysema) suggesting esophageal rupture due to Boerhaave syndrome with transhiatal herniation (Fig. 1). The patient underwent an emergent explorative operation for primary repair of the esophageal injury with VAC device insertion. However, slight but persistent drainage of gastric contents to the chest tube was noted after the thoracic operation and VAC application.

An upper GI series with Gastrografin (Bayer, Leverkusen, Germany) was performed, and a fistula tract connecting the left pleural space and primary closure site of the distal esophagus was noted (Fig. 2A). An esophagogastroduodenoscopy was performed, and a fistula orifice with mucosal defect was found at the distal esophagus (Fig. 2B). The fistula orifice diameter was approximately $<10 \mathrm{~mm}$.

Endoscopic treatment of the fistula using the "traumatic-type" OTSC system, which is designed for the closure of gastric fistula, was recommended. Written informed consent from the patient and approval of our hospital's medical scientific committee were obtained. A 30-minute endoscopic procedure using the OTSC system was performed to seal the perforation (Fig. 3). The postprocedural period was uneventful, and the amount of drainage to the chest tube was dramatically reduced. Five days after OTSC placement, a follow-up upper GI series confirmed complete sealing of the
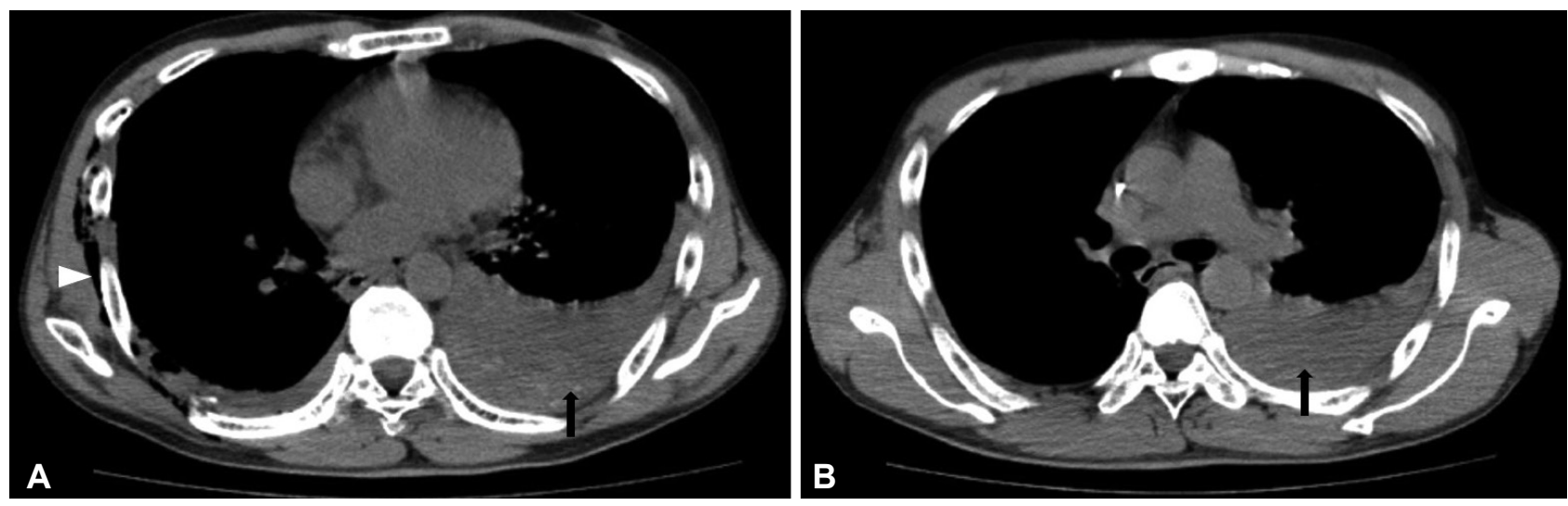

Fig. 1. Computed tomography examination shows the presence of pleuroparenchymal injury (pneumothorax, pleural effusion [black arrows], and subcutaneous emphysema [white arrowhead]), suggestive of esophageal rupture.
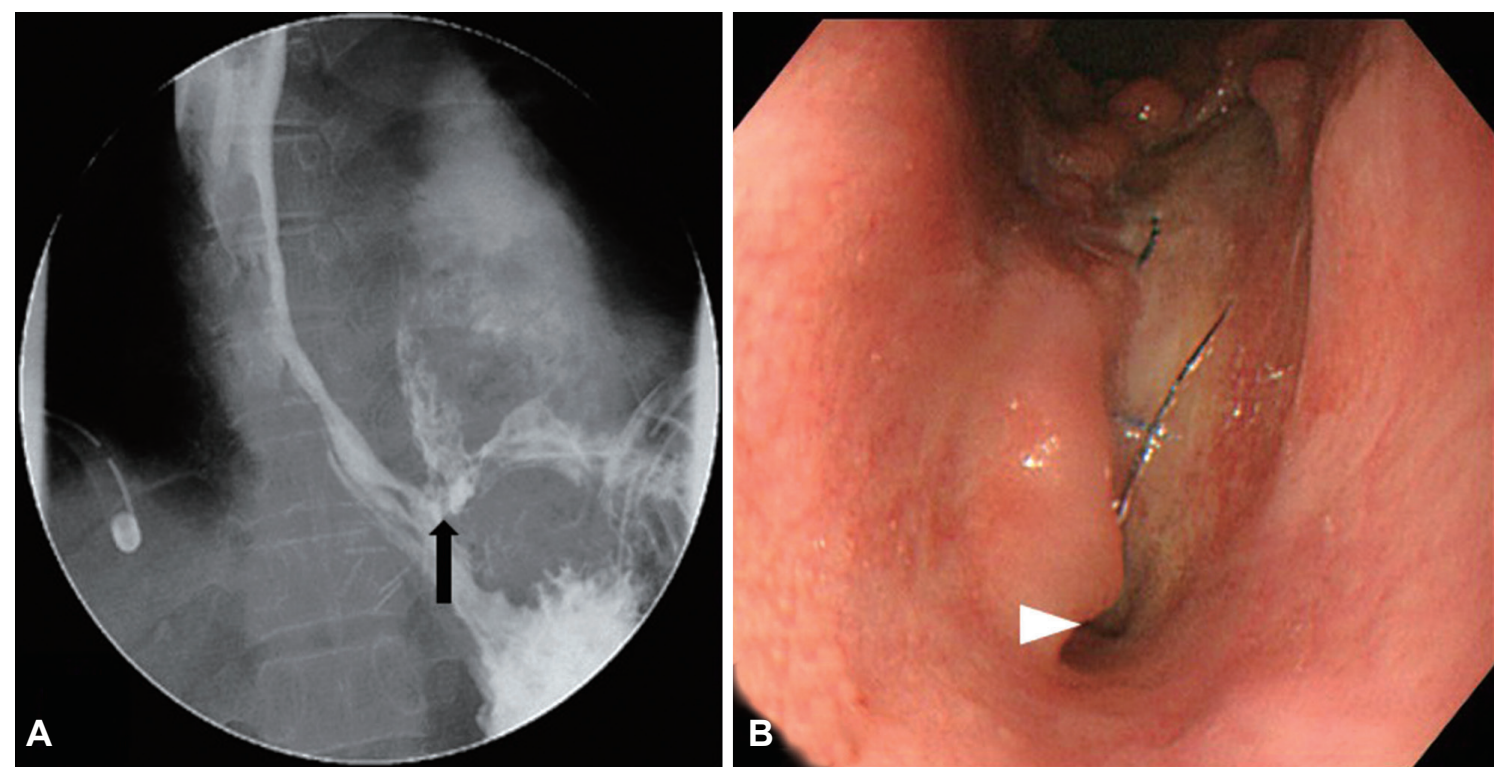

Fig. 2. Diagnosis of an esophagopleural fistula (EPF). (A) An upper gastrointestinal series with Gastrografin (Bayer) shows a fistula tract connecting the left pleural space and the closure site of the distal esophagus (black arrow). (B) Esophagogastroduodenoscopic examination reveals an esophageal orifice of the EPF at the distal esophagus (white arrowhead). 

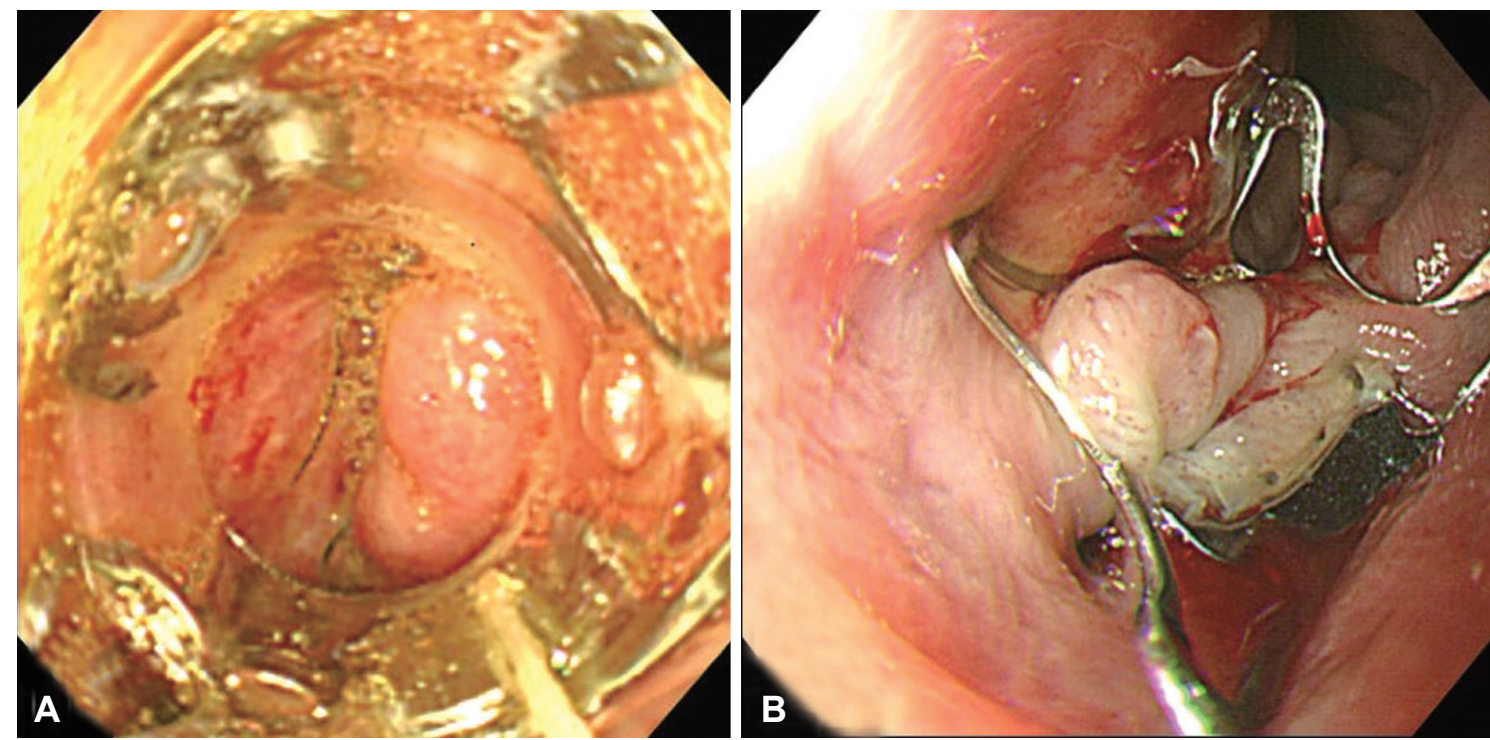

Fig. 3. Endoscopic findings during the procedure. (A) The traumatic-type over-the-scope-clip (OTSC) system is deployed at the end of the transparent application cap and approaches the esophageal orifice. (B) The OTSC system is successfully applied to the target lesion.
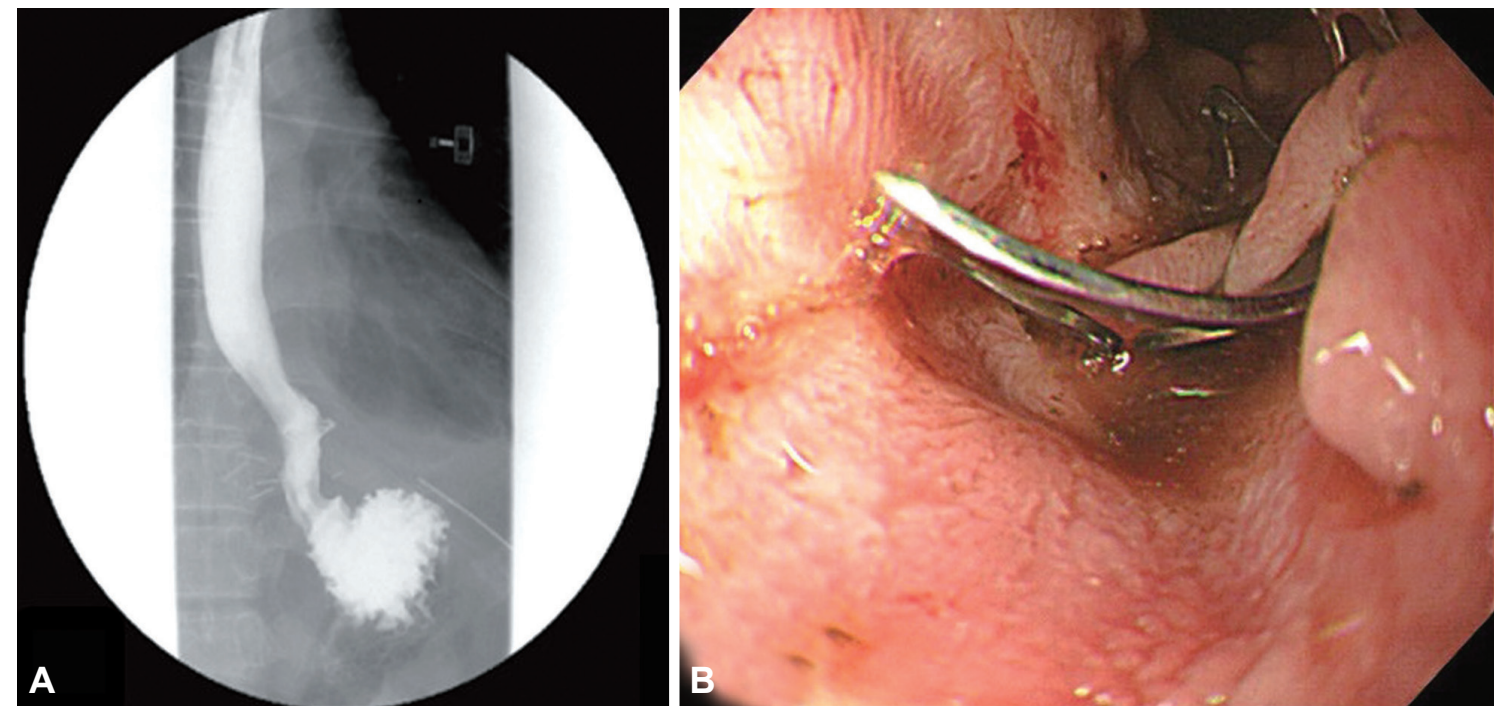

Fig. 4. Follow-up evaluation after the procedure. (A) An upper gastrointestinal series with Gastrografin (Bayer), performed 5 days after placement of the over-the scope clip system reveals no contrast leakage at the distal esophagus. (B) An esophagogastroduodenoscopic examination, performed 2 weeks postoperatively, shows that the esophageal orifice of the esophagopleural fistula at the distal esophagus has completely healed.

fistula orifice (Fig. 4A), and the patient resumed an oral diet after 7 days. After 2 weeks, a follow-up esophagogastroduodenoscopy revealed that the OTSC device was well positioned and the previous esophageal orifice of the fistula was completely healed (Fig. 4B). During the 6-month follow-up at an outpatient clinic, no OTSC-related complications were found.

\section{DISCUSSION}

Several endoscopic methods have been recently described for the treatment of esophageal or gastric fistulas, including endoscopic clipping. However, the difficulty of hemoclip application is well known, and many investigators have demanded larger, stronger clips while highlighting the fact that the available clips are difficult to apply, with repeated applications being necessary for successful treatment. The OTSC system imitates a surgical suture, and compared with conventional hemoclip systems, significantly more tissue can be grasped. Here, we describe a novel method used to close an esophageal fistula with surrounding severe fibrosis due to a previous surgery by the application of a newly developed endoscopic 
clipping system.

Currently, the main indications for OTSC application are uncontrolled primary or postinterventional bleeding in the GI tract or closure of iatrogenic full-thickness or covered perforations. Application of the OTSC yields a high success rate of endoscopic closure in patients with GI perforation and may reduce the need for emergency surgery ${ }^{10,11}$ In previous reports, Kirschniak et al. ${ }^{12}$ performed endoscopic therapy using the OTSC system in 11 patients with severe bleeding or perforation of the GI tract, and Schurr et al. ${ }^{13}$ used the OTSC clip to close the gastrostomy site after NOTES. In a more expanded indication, Aly and $\mathrm{Lim}^{14}$ showed that a gastric fistula, occurring after sleeve gastrectomy, could be successfully closed using this technique. In another recent report, Iacopini et al. ${ }^{15}$ described the closure of two chronic gastrocutaneous fistulas using the OTSC system.

There are two versions of the OTSC system: ${ }^{11}$ the "atraumatic" version (with blunt teeth) and the traumatic version (with sharp teeth). The atraumatic version is used for clipping fresh borders or vessels, while the traumatic version is effective for grasping fibrotic tissues. In this case, we chose the traumatic-type OTSC with sharp teeth to achieve the best possible grip of the fibrotic tissue around the fistulous opening. Because the OTSC system should be applied immediately after the lesion is first seen, correct fitting of the transparent application cap to the target lesion is another important technical issue for successful application of the OTSC system. To avoid inadequate visual contact at the moment of application, the endoscopist should ensure that the target is in the right position.

In our case, the patient underwent an explorative operation for primary repair of the esophageal injury with VAC device insertion before endoscopic placement of the OTSC. VAC device can effectively reduce bacterial levels and the risk of serious coinfection; furthermore, it removes extracellular fluid and contracts the wound to promote more efficient healing. However, in our case, the VAC device did not seem to be sufficient for complete healing of the fistulous tract, and slight but persistent drainage of gastric contents to the chest tube was noted after VAC application. Our results suggest that the OTSC system might be a good alternative method for the management of complicated EPFs or other enteric fistulas refractory to VAC devices.

In conclusion, the OTSC system might represent a novel and promising modality for EPF treatment, and it is safe and effective compared to established endoscopic or surgical techniques. To extend the classic indications of the OTSC system in interventional GI endoscopy, prospective comparative studies will be necessary to confirm the sufficiency and efficacy of this new method for the closure of esophageal or gastric fistulas.

\section{Conflicts of Interest}

The authors have no financial conflicts of interest.

\section{REFERENCES}

1. Khamaysi I, Suissa A, Yassin K, Gralnek IM. Closure of a persistent esophagopleural fistula using an over-the-scope clipping device. Endoscopy 2013;45 Suppl 2 UCTN:E29-E30.

2. Orgill DP, Manders EK, Sumpio BE, et al. The mechanisms of action of vacuum assisted closure: more to learn. Surgery 2009;146:40-51.

3. Lambert KV, Hayes P, McCarthy M. Vacuum assisted closure: a review of development and current applications. Eur J Vasc Endovasc Surg 2005;29:219-226.

4. Cro C, George KJ, Donnelly J, Irwin ST, Gardiner KR. Vacuum assisted closure system in the management of enterocutaneous fistulae. Postgrad Med J 2002;78:364-365.

5. Sandmann M, Heike M, Faehndrich M. Application of the OTSC system for the closure of fistulas, anastomosal leakages and perforations within the gastrointestinal tract. Z Gastroenterol 2011;49:981-985.

6. Nishiyama N, Mori H, Kobara H, et al. Efficacy and safety of over-thescope clip: including complications after endoscopic submucosal dissection. World J Gastroenterol 2013;19:2752-2760.

7. Mennigen R, Colombo-Benkmann M, Senninger N, Laukoetter M. Endoscopic closure of postoperative gastrointestinal leakages and fistulas with the Over-the-Scope Clip (OTSC). J Gastrointest Surg 2013;17:10581065.

8. Singhal S, Changela K, Papafragkakis H, Anand S, Krishnaiah M, Duddempudi S. Over the scope clip: technique and expanding clinical applications. J Clin Gastroenterol 2013;47:749-756.

9. Dişibeyaz S, Köksal AS, Parlak E, Torun S, Şaşmaz N. Endoscopic closure of gastrointestinal defects with an over-the-scope clip device. A case series and review of the literature. Clin Res Hepatol Gastroenterol 2012;36:614-621.

10. Hagel AF, Naegel A, Lindner AS, et al. Over-the-scope clip application yields a high rate of closure in gastrointestinal perforations and may reduce emergency surgery. J Gastrointest Surg 2012;16:2132-2138.

11. Weiland T, Fehlker M, Gottwald T, Schurr MO. Performance of the OTSC System in the endoscopic closure of iatrogenic gastrointestinal perforations: a systematic review. Surg Endosc 2013;27:2258-2274.

12. Kirschniak A, Kratt T, Stüker D, Braun A, Schurr MO, Königsrainer A. A new endoscopic over-the-scope clip system for treatment of lesions and bleeding in the GI tract: first clinical experiences. Gastrointest Endosc 2007;66:162-167.

13. Schurr MO, Arezzo A, Ho CN, Anhoeck G, Buess G, Di Lorenzo N. The OTSC clip for endoscopic organ closure in NOTES: device and technique. Minim Invasive Ther Allied Technol 2008;17:262-266.

14. Aly A, Lim HK. The use of over the scope clip (OTSC) device for sleeve gastrectomy leak. J Gastrointest Surg 2013;17:606-608.

15. Iacopini F, Di Lorenzo N, Altorio F, Schurr MO, Scozzarro A. Over-thescope clip closure of two chronic fistulas after gastric band penetration. World J Gastroenterol 2010;16:1665-1669. 